\title{
An Improved LDV-Hop Localization Algorithm in Wireless Sensor Networks
}

\author{
Xiaoan Bao, Zhongtao Ren, Na Zhang, Xing Hu, Tongshu Wu and Yangqiu Liu \\ The institute of software of Zhejiang Sci-Tech University \\ baoxiaoan@zstu.edu.cn
}

\begin{abstract}
Localization of sensor nodes is a fundamental and a key technology in wireless sensor networks, it has been widely studied by many universities and research institutions in recent years and many localization algorithms have been put forward by scholars from diverse aspects. A new localization algorithm, which is based on the LDV-Hop algorithm, is proposed in this paper. Firstly, to reduce the localization error we modify the average one-hop distance between anchor nodes, and the average one-hop distance used for each unknown node's location is also modified through weighting the received average one-hop distance from anchor nodes. Then, to solve the problem of low localization coverage which is the main disadvantage of LDV-Hop localization algorithm, we regard regular node with newly estimated location as anchor node and utilize believable factor threshold to restrain the error accumulation brought by this method.
\end{abstract}

Keywords: Localization; wireless sensor networks; localization coverage; believable factor threshold

\section{Introduction}

Wireless sensor networks consist of plenty of inexpensive smart nodes. Just like a small computing device, each node has the capability of perception, processing and communication with a power source on-board. WSNs have wide applications in military, medical and many other areas such as hospital setting, monitoring applications [1, 2], tagging small animals, machine diagnosis and other intelligent environments [3]. In these large sensor networks, we need to know nodes' location. But manual configuration of location is infeasible, and providing each node with localization hardware is expensive in terms of cost and energy consumption and simply unavailable in many deployment scenarios. This problem is a challenging and extremely crucial.

In recent years many node localization methods have been put forward by universities and research institutions, and these algorithms are divided into two categories: range-based and range-free [4]. The former is based on measuring the distance or angle information between neighbor nodes, and uses the trilateral or multilateral localization method to gain precise node location information. But range-based algorithms require the sensor nodes equip additional hardware. The latter is based on network connectivity information such as Weighted Centroid Algorithm [5], DV-Hop algorithm and APIT. Though range-free localization algorithm provides just coarse granularity information, it does not require additional hardware devices and its location accuracy is enough in most applications. So range-free localization algorithms have been more widely used in wireless sensor networks.

In this paper, we propose an improved localization algorithm to improve the low localization coverage in DV-Hop localization algorithm which has attractive advantages of 
low localization error and low computation and communication cost. The rest of the paper is organized as follows. In Section 2, we describe the basic DV-Hop algorithm and some existing improved DV-Hop algorithms. In Section 3, our algorithm will be depicted. In Section 4, the simulation results are presented and analyzed. Finally, we will give the conclusion in Section 5.

\section{Related works}

\subsection{DV-Hop Localization Algorithm}

DV-Hop localization algorithm (distance vector hop) [6] was proposed by Dragos Niculescu from Rutgers University as one of the typical representatives of range-free localization algorithm, the algorithm implementation consists of three steps.

In the first step, each node computes the shortest hops to the anchor nodes through typical Distance Vector Routing. In the second step, once an anchor gets hop count value to all the other anchor nodes and the practical distance between them, it can calculate the average hop size which is the average ratio of distance and hop count to all the other anchor nodes. The average hop size is estimated by the th anchor node using the following formula:

$$
\text { HopSize }_{i}=\frac{\sum_{i \neq j} \sqrt{\left(x_{i}-x_{j}\right)^{2}+\left(y_{i}-y_{j}\right)^{2}}}{\sum_{i \neq j} h_{o p}}
$$

Where $\left(x_{i}, y_{i}\right)$ and $\left(x_{j}, y_{j}\right)$ are the coordinates of anchor nodes $i$ and $j$ respectively, and $h o p_{i j}$ is the hop count between them. Then it propagates the hop size to the entire network. After receiving the hop size, the regular nodes estimate its distance from the anchor node by multiplying the hop size by the hop count value.

However, regular nodes receive the hop size information but just save the first one, ignoring others, and transmit it to their neighbor nodes simultaneously. This method ensures nodes can receive the hop size from the anchor node which has the least hops between them. In the last step, when regular nodes get three or more distance information from anchor nodes, we can calculate the locations by trilateral measurement or the maximum likelihood estimation method.

\subsection{Some Existing Improved DV-Hop Algorithm}

DV-Hop localization algorithm is simple but it could achieve proper location accuracy when nodes are distributed uniform and isotropic in WSN. However its accuracy would reduce rapidly with the increase of distance error, and when the nodes are deployed unevenly, they often receive inaccurate hop information and calculate the hop size with bigger error, which could badly affect the location accuracy.

To solve this problem, many improved DV-Hop localization algorithms had been proposed from various aspects. To increase the location accuracy, Guha et al., [7] propose an accurate, cheap and scalable framework, called Sextant, which operates by setting up and solving a system of geographic constraints based on connectivity information from the underlying communication network. Kaoru Sezaki2 et al., [8] uses 2-D Hyperbolic localization algorithm and a least square method in step 3. Shi used the cluster strategy to reduce the communication cost, and group the conflict probability in the first stage. Then he used the quasi-Newton optimization algorithm to estimate the location of nodes, which improved the localization accuracy without increasing communications traffic [9]. To increase the location accuracy of sensor network with imprecise distance, Cao et al describe the geometric relations among the 
sensor-anchor distances as one or multiple quadratic equality constraints by using the CayleyMenger determinant [10]. The goal of the sensor network localization problem is to determine positions of all the sensor nodes in a network given certain wise noisy distance measurements and inaccurate anchor node positions. A two-step distributed localization approach based on second-order cone programming (SOCP) relaxation is presented in literature [11] by Srirangarajan and Seshan. In the first step, the sensor nodes determine their positions based on local information and in the second step, the anchor nodes refine their positions using information from the neighboring nodes. The second step enables anchors which are in the convex hull of their neighbors to refine their positions. H Chen, K Sezaki et al. [12] improve a new localization algorithm based on the DV-Hop localization algorithm, they focus on the step 2 and step3 of DV-Hop localization algorithm. In step 2, they average whole of the hopsize of different anchor nodes, and those unknown nodes would compute the distance to the beacon nodes based hop-length and hops to the beacon nodes. In step 3, they develop a general model of two dimensional position location estimation of a source using $\mathrm{M}$ anchor nodes. They do not adopt traditional Triangulation algorithm but use two dimensional Hyperbolic location algorithm $[13,14]$.

\subsection{The Limit DV-Hop Localization Algorithm}

The Limit DV-Hop localization algorithm is aimed to accomplish node location in limited areas through adjusting the amount of receiving packets in node location processing. Specifically, through setting a threshold $\mathrm{N}$ the regular nodes refuse to receive the packet transmitted from node whose hop count value is greater than $\mathrm{N}$. The steps of this method are as follows.

Firstly, anchor nodes periodically broadcast a beacon message, which contains hops, its location and its ID. The unknown nodes receive the beacon message from certain an anchor node, but just save the packet with shortest hop value and ignore the other packets with larger hop value from the same anchor. Then it compares the recorded shortest hop value to the set threshold $\mathrm{N}$ : if the recorded shortest hop value is less than $\mathrm{N}$, the shortest hop value would be added to 1 and then the packet is broadcast to neighbor nodes. Otherwise, the packet is abandoned and refused to transmit.

Secondly, utilizing the formula.1, each anchor computes the Hop Size with the recorded hop count value between other anchor nodes and these anchor nodes' location information, then broadcasts the result to the network. The unknown nodes receive $t$ and record the first Hop Size they received, which ensures that each unknown node could get the Hop Size from the nearest anchor in hops.

Finally, we can use the trilateral measuring method or the maximum likelihood estimation method to compute the estimate location of regular nodes.

This localization algorithm not only can substantially increase the location accuracy, but also vastly reduce the communication cost. But in reality, the network topology is not uniformly distributed in area which is hard to control and affects the localization error badly. In literature [15], the author proves that when beacon nodes are collinear or nearly collinear, even a tiny measurement would cause a big localization error. So it's necessary to judge the collinear degree of beacon nodes which are within the given hops in step 1. 


\section{A New Improved Algorithm Based on LDV-Hop}

\subsection{Modify the Calculation of the Average Hop Distance of Unknown Nodes}

$$
\text { HopSize }_{i}=\frac{\sum_{i \neq j} \text { hop }_{i j} d_{i j}}{\sum_{i \neq j} \text { hop }_{i j}^{2}}
$$

Where $d_{i j}$ is the practical distance between the anchor node $i$ with $j$.

Then, we modify the average hop distance ( Hop Size $_{u}$ ) of regular nodes through weighting received average hop distances from the $\mathrm{n}$ anchor nodes by following formula:

$$
\delta=\left\{\begin{array}{l}
1, \text { anchor node } \\
1-\frac{e}{\sum_{i} \sqrt{\left(x^{\prime}-x_{i}\right)^{2}+\left(y^{\prime}-y_{i}\right)^{2}}} \text {, regular nodes }
\end{array}\right.
$$

The total of weighting value $\lambda_{i}$ of all anchors in the routing table of unknown nodes is 1 . By the average, the average hop distance of unknown nodes is reflected from the average hop distance of all anchor nodes.

\subsection{The New Improved Algorithm}

Limit DV-Hop localization algorithm, which accomplishes node localization in limited areas, could increase the localization accuracy obviously and reduce communication cost vastly at the same time. Besides, this algorithm is also adaptive to the networks whose nodes are distributed irregularly. But the node localization coverage is too low especially in the large networks with low proportion of anchor nodes and low node connectivity.

However, in order to reduce costs, anchor nodes always are distributed as small as possible, which is bound to reduce node localization coverage if we only use LDV-Hop localization algorithm. To solve this problem, the new algorithm inherits advantages of LDVHop localization algorithm, and regard nodes with newly estimated location as an anchor node. In such a way the majority of the nodes can finish their localization. But this technology will propagate the localization error from those newly estimate position nodes as the localization process, which means that the inaccurate location estimate of one node could be used by other nodes to estimate their own location, increasing localization inaccuracy undoubtedly. Location error caused by error accumulation will be unable to accept. Only when the location accuracy is high enough, can regular nodes be regarded as new anchor nodes. Ref [17] used the non-linear least squares technique to estimate the coordinates of regular nodes, which is minimizing the residual error. In the new algorithm, location error is estimated as following formula:

$$
\lambda_{i}=\frac{\text { hop }_{i}}{\sum_{k=1}^{n} \text { hop }_{k}} \quad \text { HopSize }_{u}=\sum_{i=1}^{n} \lambda_{i} \text { HopSize }_{i}
$$

Where $\left(x^{\prime}, y^{\prime}\right)$ is the estimated coordinate of the regular node, $\left(x_{i}, y_{i}\right)$ is the $i$ th anchor node's location, $d_{i}$ is the measured distance between the regular node and $i$ th anchor node.

In order to alleviate the error propagation effect, each node with known location has a believable factor $\delta$ which is calculated as follows:

$$
e=\sum_{i}\left\{\sqrt{\left(x^{\prime}-x_{i}\right)^{2}+\left(y^{\prime}-y_{i}\right)^{2}}-d_{i}\right)
$$


According to the localization accuracy requirement, a threshold $\lambda$ is set. For the anchor nodes, $\delta$ value is set to 1 . While for regular nodes, $\delta$ value is associated with its location error, which can be simply calculated by the formula (5). And as we can see, the bigger the location error is, the smaller the believable factor $\delta$ is. When $\delta>\lambda$, the regular finishes its localization and can be regarded as a new anchor node. Otherwise it will continue to be nonlocalized.

\subsection{Process of the New Improved Algorithm}

In the initialization phase, all anchor nodes set their $\delta$ value to 1 . All regular nodes are still non-localized nodes. With the advance of localization process, more and more regular nodes get their location estimated and become anchor nodes, and then they broadcast their localization message, which contains coordinate, their ID and believable factor value, to neighbor nodes.

Considering the two-dimensional case, we assume that in certain a limit area, regular node $i$ th can estimate the distance to $\mathrm{N}$ anchor nodes which include newly estimated location nodes.

Firstly, if $\mathrm{N}<3$, the localization process has to wait until enough unknown nodes get their estimated precise location by using the distance measurements to three nearest anchor nodes which are in the neighbor areas.

Secondly, when $\mathrm{N}<3$, we can get all the combinations which contain three distance measurements from all the $\mathrm{N}$ distance. Assume the number of the combinations be $\mathrm{K}$, obviously $\mathrm{K}=C_{N}^{3}$. Then compute the location error e of each combination. In this step, we could get $\mathrm{K}$ location solutions, $\left(x^{\prime}{ }_{j}, y_{j}^{\prime}\right), \mathrm{j}=1,2 \ldots, \mathrm{K}$.

Finally, to compute the coordinate of $i$ th node, we have to calculate its believable factor value $\delta_{j}$ for each location solution in second step at first. Then we use Weighted Centroid Algorithm to get the final location estimate of $i$ th node:

$$
\left(x_{i}, y_{i}^{\prime}\right)=\left(\frac{\sum_{j=1}^{K} \delta j_{j} x_{j}^{\prime}{ }_{j}^{K}}{\sum_{j=1}^{K} \delta_{j}}, \frac{\sum_{j=1}^{K} \delta_{j} y_{j}^{\prime}}{\sum_{j=1}^{K} \delta_{j}}\right)
$$

Fourthly, the regular node computes its believable factor $\delta$ after it gets its estimated location. If $\delta \geq \lambda$, the regular node is localized and labels itself as a new anchor node. Otherwise, the node will repeat the first three steps. However, if node always can't get the location which meets the requirement, it will endlessly broadcast localization message which should be avoided. Assume that $\delta_{n}$ denotes node's believable factor value in the nth round, if the difference between $\delta_{n}$ and $\delta_{n+1}$ is smaller than a small constant $\eta(\eta=0.01)$, the algorithm will be over.

The localization Algorithm could greatly increase node localization coverage and keep those attractive advantages of LDV-Hop localization algorithm.

\section{Simulation}

In order to evaluate our algorithm, we ran many experiments on DV-Hop, LDV-Hop and the improved LDV-Hop algorithms using the NS2 simulation environment. 100 nodes are randomly distributed in a $150 \mathrm{~m} \times 150 \mathrm{~m}$ region and the transmission range is ranged to be $5 \mathrm{~m}$. 
We consider two performance metrics: localization accuracy and localization coverage in the different number of anchor nodes and believable factor threshold.

\subsection{Impact of the Number of Anchor Nodes}

In this set of simulations, the believable factor threshold $\delta$ is set as 0.8 , and the threshold $\mathrm{N}$ in LDV-Hop algorithm is set to be 8. We change the number of anchor nodes from 10 to 80 , comparing the localization error and coverage of these three algorithms. The results are shown in Figure 1.
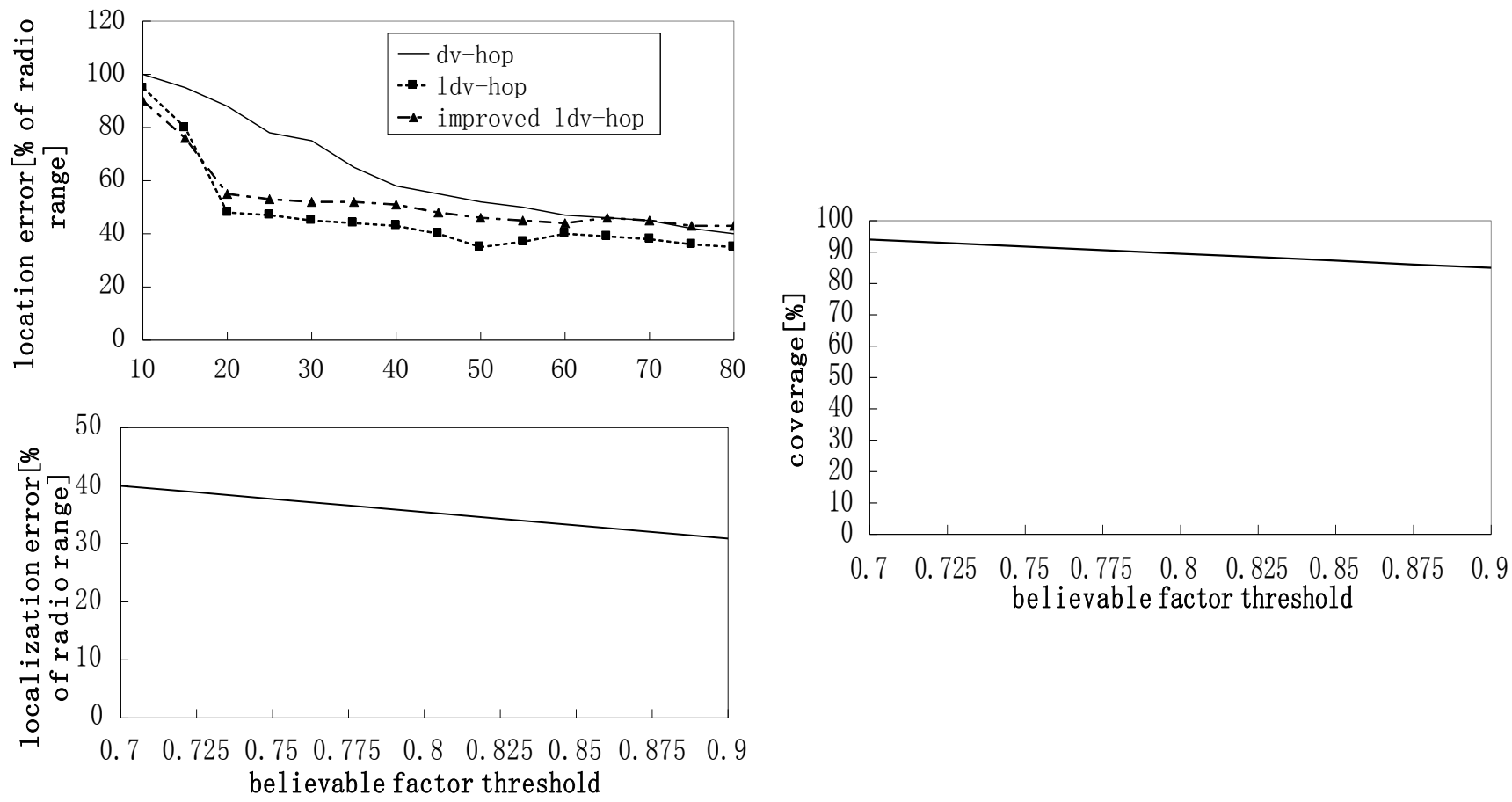

Figure 1. Impact of the Number of Anchor Nodes

The localization error and coverage with different numbers of anchor nodes are shown in Figure 1. As we see, the localization error in Limit DV-Hop localization algorithm is almost $40 \%$ lower than that in DV-Hop algorithm when the anchor node radio is about $20 \%-50 \%$, and the localization error in our algorithm is slightly higher than that in LDV-Hop algorithm, this is because we can't eliminate the error caused by location error propagated from those newly estimate position nodes as the localization process, but it is so little that we don't need to worry about this disadvantage. From the second figure, we see clearly that in the sensor network with low node connectivity, which is lower than 5 in our simulation environment, the coverage is very low in the DV-Hop localization algorithm. What is worse is that coverage is even lower in the Limit DV-Hop algorithm, this is because we set a threshold $\mathrm{N}(\mathrm{N}=8$ in our simulation environment) in LDV-Hop algorithm, which makes the unknown nodes refuse to receive the beacon messages from the anchor nodes which are far away in hops. While, utilizing the location information of those regular nodes with newly estimated precise position makes the coverage increase about more than $40 \%$ in our algorithm. That is just what we expect. 


\subsection{Impact of Believable Factor Threshold}

Here, we set the number of anchor node is 40 and change the believable factor threshold $\delta$ from 0.7 to 0.9 . The simulation results are shown in Figure 2.

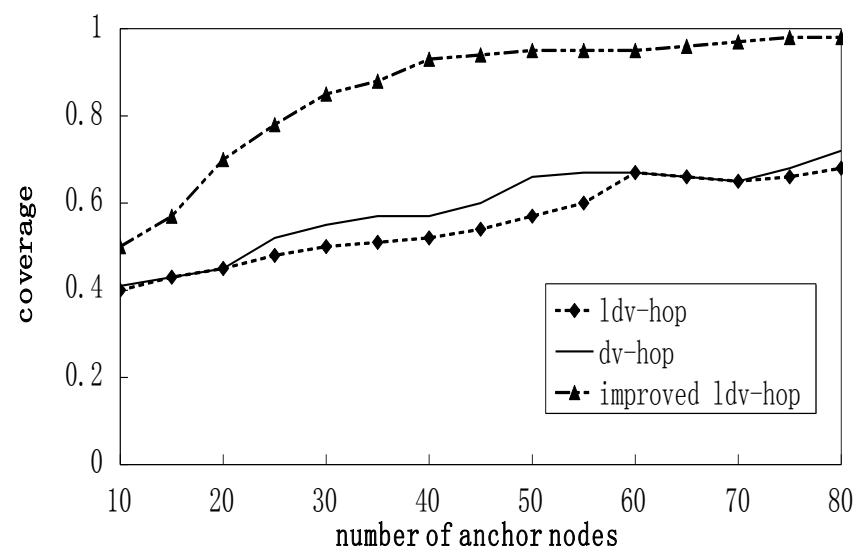

Figure 2. Impact of Believable Factor Threshold

Figure 2 shows that the localization error and coverage will decrease a little with the increase of $\delta$ value. This is because the higher the $\delta$ value is, the higher location precision will the anchor nodes participating in the localization process have, which will decrease the localization error undoubtedly. But at the same time, the localizable nodes will be reduced and the localization coverage will be reduced as well. Besides, it proves us that we can control the compromise between localization accuracy and coverage by changing the $\delta$ value. For example, for those WSNs which only require high localization coverage rather than localization accuracy, the believable factor threshold should be set to a relatively small value.

\section{Conclusion}

In this paper, we propose a improved LDV-Hop localization algorithm. It not only keeps the advantages of high accuracy and attractively low computation cost and low communication cost, but also overcomes the defect of low localization coverage in Limit DVHop algorithm by using

Regular nodes with newly estimated precise location as anchor nodes and setting a believable factor threshold to reduce location error accumulation. The simulation results show our algorithm can be well applied to many wireless sensor networks with low connectivity.

\section{Acknowledgements}

This work was supported by National Natural Science Foundation of China (no. 61202050, no. 61170325, no. 61379036), Zhejiang Provincial Natural Science Foundation of China (LY12F02041, Y13F020175), Zhejiang Qianjiang Talent Project(2013R10015, 2013R10005), the New-shoot Talents Program of Zhejiang province(2012R406071, 2014R406073), and the project of training high skilled talents in Zhejiang Province(2013R30001, 2013R30019). 


\section{References}

[1] G. R. Hayes, D. J. Patterson, M. Singh, D. Gravem, "Supporting the transition from hospital to home for premature infants using integrated mobile computing and sensor support", Pers Ubiquit Comput, vol. 15, no. 8, (2011), pp. 871-88.

[2] J. P. Varkey, D. Pompili and T. A. Walls, "Human motion recognition using a wireless sensor-based wearable system", Pers Ubiquit Comput, vol. 16, no. 7, (2012), pp. 897-910.

[3] X. Hong and C. D. Nugent, "Segmenting sensor data for activity monitoring in smart environments", Pers Ubiquit Comput, vol. 17, no. 3, (2013), pp. 545-559.

[4] T. He, C. D. Huang, B. M. Blum, J. A. Stankovic and T. Abdelzaher, "Range-Free localization schemes in large scale sensor networks", In: Proc.of the 9th Annual Int'1 Conf.on Mobile Computing and Networking. San Diego, ACM Press, (2003), pp. 81-95.

[5] B. Li and H. Wang, "A Low Complexity Localization Algorithm In Wireless Sensor Network, 2010 International Conference on Innovative Computing and Communication and 2010 Asia-Pacific Conference on Information Technology and Ocean Engineering, (2010).

[6] J. Kurose and K. Ross, "Computer Networks: A Top Down Approach Featuring the Internet", [M], Pearson Addison Wesley, (2006).

[7] S. Guha, R. N. Murty and E. G. Sirer, "Sextant: A unified node and event localization framework using nonconvex constraints", In: Proceedings of the 6th ACM International Symposium on Mobile Ad Hoc Networking and Computing, IL, USA, (2005), pp. 205-216.

[8] H. Y. Chen, K. Sezaki, P. Deng and H. C. So, "An Improved DV-Hop Localization Algorithm for Wireless Sensor Works", Industrial Electronics and Applications, (2008).

[9] S. Hongyu and Y. Sha, "An improved DV- Hop localization algorithm for wireless sensor network", Electronics Optics \& Control, vol. 18, no. 4, (2011), pp. 93-96.

[10] M. Cao, B. Anderson, A. S. Morse, "Sensor network localization with imprecise distances", Systems \& control letters, (2006).

[11] S. Seshan, "Distributed Sensor Network Localization with Inaccurate Anchor Positions and Noisy Distance Information", In: IEEE International Conference on Aeousties, Speech and Signal Processing,( 2007), III521 - III524.

[12] H. Chen, K. Sezaki and P. Deng, et al, "An improved DV-Hop localization algorithm for wireless sensor networks", [C], Industrial Electronics and Applications, 2008, ICIEA 2008, 3rd IEEE Conference on, IEEE, (2008), pp. 1557-1561.

[13] D. J. Torrieri, "Statistical theory of passive location systems", [J], Aerospace and Electronic Systems, IEEE Transactions on, no. 2, (1984), pp. 183-198.

[14] Y. T. Chan and K. C. Ho, "A simple and efficient estimator for hyperbolic location", [J], Signal Processing, IEEE Transactions on, vol. 42, no. 8, (1994), pp. 1905-1915.

[15] C. Bettstetter, "On the minimum node degree and connectivity of a wireless multihop network", [C], International Symposium on Mobile Ad Hoc Networking \& Computing: Proceedings of the 3 rd ACM international symposium on Mobile ad hoc networking \& computing, vol. 9, no. 11. (2002), pp. 80-91.

[16] J. Z. Lin, X. B. Chen and H. B. Liu, "Iterative algorithm for locating nodes in WSN based on modifying average hopping distances", Journal on Communications, vol. 30, no. 10, (2009), pp.107-113.

[17] S. Gezici, "A survey on wireless position estimation, Springer Wireless Personal Communications", Special Issue Towards Global and Seamless Personal Navigation, (2007) Oct. 


\begin{tabular}{|c|c|c|c|}
\hline 论文题目 & An Improved LDV-Hop Localization Algorithm in Wireless Sensor \\
Networks
\end{tabular}


International Journal of Control and Automation Vol.7, No.10 (2014) 